WaveOne Gold

sisteminin farklı rehber

yol varlığı ve

yokluğundaki

şekillendirme

etkinliğinin S şeklinde

yapay kanallar üzerinde

karşılaştırılması

\section{Comparison of the shaping ability of WaveOne Gold system with or without different glide path techniques in simulated curved S-shaped root canals}

\section{Dr. Ayca Yilmaz}

İstanbul Üniversitesi, Diş Hekimliği Fakültesi, Endodonti A.D., İstanbul

Orcid ID: 0000-0002-9254-7710

Geliș tarihi: 2 Eylül 2019

Kabul tarihi: 9 Aralık 2019

doi: 10.5505/yeditepe.2020.22438

\section{Yazışma adresi:}

Dr. Ayça Yılmaz

Göktürk Merkez Mah. Göktürk Cad. No.30 B2 D7

Eyüp/istanbul

Tel: 00905325163400

E-mail: aycasimsek@gmail.com
ÖZET

Amaç: Bu çalışmanın amacl, rotasyon ve resiprokasyon hareketleriyle çalışan rehber aletler varlığı ve yokluğunda WaveOne Gold sisteminin şekillendirme etkinliğinin S şeklindeki yapay kanallar üzerinde karşılaştırılmasıdır.

Gereç ve Yöntem: Çalışmada 45 adet S-kanallı, 0.02 koniklik açılı, ISO 15 rezin blok kullanılmıştır. Bloklar numaralandırılmış, yapay kanallar siyah mürekkeple boyanmıştır. Şekillendirme öncesi bloklar taranarak görüntüler kaydedilmiştir. Bloklar şekillendirme yöntemine göre 3 gruba ayrılmıştır ( $n=15)$, Grup A: WaveOne Gold (Primary, 25/.07), Grup B: ProGlider-WaveOne Gold ve Grup C: WaveOne Gold Glider-WaveOne Gold. Bütün bloklar şekillendirme sonrası kırmızı mürekkep ile boyanmış ve tekrar taranarak görüntüler kaydedilmiştir. Yapay kanalların işlem öncesi ve sonrası görüntüleri imaj analiz programı yardımıyla çakıştııımıştır. Kanalların iç ve dış bölgesinden kaldırılan rezin miktaları çakıştırılan görüntüler üzerinden belirlenen 12 noktadan bilgisayar programı yardımıyla ölçülmüş, grupların şekillendirme etkinlikleri kanal transportasyonu ve merkezleme oranları açısından karşılaştırılmıştır. Veriler Shapiro-Wilk normallik testi, Kruskal Wallis ve Dunn'un çoklu karşılaştırma testi kullanılarak istatistiki olarak değerlendirilmiştir.

Bulgular: Karşılaştıılan kriterler açısından düz kuronal ve birinci eğim bölgesinde gruplar arasında istatistiki olarak anlamlı farklılık bulunmamıştır $(p>0,05)$. Apikal eğim bölgesinde ise $A$ grubunun transportasyon değerleri $B$ ve $C$ grubundan istatistiki olarak anlamlı yüksek bulunmuştur ( $p=0,002, p=0,011$ ). Merkezleme değerleri karşılaştırıldığında, $C$ grubunun apikkal eğim bölgesindeki merkezleme değerleri $A$ ve $B$ grubunun değerlerinden istatistiksel olarak anlamlı yüksek bulunmuştur $(p=0,001$, $\mathrm{p}=0,001$ ).

Sonuç: Bu çalışmanın koşulları altında değerlendirildiğinde, WaneOne Gold sistemiyle S kanalların şekillendirilmesinde öncelikle resiprokasyon yapan WaveOne Gold Glider ile giriş yolu oluşturulduğunda apikal bölgede daha merkezi bir şekillendirme elde edilmektedir. Wave One Gold sistemi öncesi rehber yol oluşturmak apikal bölgedeki transportasyon miktarını azaltmaktadır.

Anahtar kelimeler: Merkezleme oranı, nikel titanyum, rehber yol, resiprokasyon, transportasyon.

\section{SUMMARY}

Aim: The aim of the present study was to compare the shaping ability of WaveOne Gold instruments with or without diffferent glide path preparation in simulated curved S-shaped canals.

Materials and Methods: Fortyfive 0.02 taper, ISO 15 resin endo training S-blocks were studied. The simulated canals were dyed using black ink. Blocks were scanned and preinstrumentation images were reccorded. The blocks were divided into 3 groups $(n=15)$ according to the instru- 
mentation protocole as, Group A: WaveOne Gold (Primary, 25/.07), Group B: ProGlider-WaveOne Gold and Group C: WaveOne Gold Glider-WaveOne Gold. All canals were then dyed again using red ink and scanned. Pre- and post-operative images were superimposed and evaluated at 12 defined points. The efficacy of the systems was compared based on the amount of canal transportation and centering ability. Data were statistically analyzed using the Shapiro-Wilk, Kruskal-Wallis and Dunn's tests.

Results: The transportation values at the apical curvature zone was statistically significantly higher for Group A than Groups B anc C ( $p=0.002, p=0.011)$. Group $C$ had significantly greater centering ability at the apical curvature zone $(p=0.001, p=0.001)$. No statistically significant difference in the amount of transportation and centering ratio at the coronal straight and first curvature zones for the groups ( $p>0.05)$.

Conclusion: Within the limitations of this study, the glide path preparation using the reciprocating WaveOne Gold Glider improved the centering ability of WaveOne Gold system and the creation of a glide path yielded better results of canal transportation than using WaveOne Gold alone at the apical curvature zone of S-shaped canals.

Keywords: Canal transportation, centering ability, glide path, nickel-titanium, reciprocation.

\section{Giriş}

Kök kanal tedavisinin başarısı temel olarak, şekillendirme, dezenfeksiyon ve doldurma adımlarının doğru uygulanmasıyla ilişkilidir. Kanal tedavisi işleminin ilk basamağı olan şekillendirme işlemi, sonraki aşamaların başarısını da direk olarak etkilemektedir. Şekillendirme sırasında kanalın orijinal şekline olabildiğince sadık kalabilmek, endodontik tedavilerde daha başarılı sonuçlara ulaşılmasını sağlamaktadır. ${ }^{1}$

Nikel titanyum (Ni-Ti) alet sistemlerinin, daha az transportasyona neden olarak daha merkezi şekillendirmeler oluşturduğu ve böylece eğri kanallarda başarılı ve etkin olarak kullanılabildikleri gösterilmiştir. ${ }^{2} \mathrm{Ni}$-Ti döner aletlerde döngüsel ve bükülme yorgunluğu sonucu oluşan alet kırıkları riskini ${ }^{3,4}$ ve aletlerin kanala vidalanma etkisini azaltmak ayrıca aletlerin merkezleme oranını arttırmak amacıyla ${ }^{5,6}$ resiprokasyon hareketiyle kullanılan $\mathrm{Ni}$-Ti sistemler kullanılmaya başlanmıştır. WaveOne Gold (Dentsply Maillefer, Ballaigues, İsviçre) sistemi resiprokasyon hareketiyle çalışan ve tek aletle şekillendirmeyedayanangüncel birsistemdir. Farklıkanallar için farklı boyutta 4 kanal aleti içerir (Small (20/.07), Primary (25/.07), Medium (35/.06), Large (45/.05)). Aletler üzerindeki koniklik açıları değişkendir. Aletlerin kesiti 2'si aktif, 4 kesici kenar içeren paralelogramdır. $7 \mathrm{Ni}$-Ti üzerine uygulanan tekrarlanan ısıtma ve soğutma ısı işlemi sonucu yüzeyde titanyum oksit tabakasının kalınlığı değişmektedir. Bu işlem sonucu bu sistemin aletleri 100- 140nm titanium oksit kalınlığıyla hafıza kontrollü gold özelliği göstermektedir. ${ }^{7}$ Bu da sistemin aletlerinin esnekliğini ve dönme yorgunluğuna dirençlerini arttırmaktadır. ${ }^{8}$ Şekillendirme işleminin daha güvenli yapılabilmesi için rehber yol oluşturulması önerilmektedir. ${ }^{9}$ Böylece şekillendirme sırasında oluşabilecek hataların, alet kırılmalarının ve aletlerin kanalda maruz kaldığı vidalanma etkisinin azaldığı gösterilmiştir. ${ }^{10-13} \mathrm{Bu}$ amaçla genellikle bütün sistemlerin kullanımından önce 10 numaralı K-tipi paslanmaz çelik el aletiyle kanala girilmesi önerilmektedir. El aletlerini takiben kullanılacak rehber yol için özel olarak üretilmiş Ni-Ti sistemler de mevcuttur. ProGlider (Dentsply Maillefer) ve WaveOne Gold Glider (Dentsply Maillefer) rehber yol için üretilmiş tek alet içeren sistemlerdir. ProGlider, M-wire Ni-Ti'dan üretilmiş, rotasyon hareketi ile çalışan, değişken konik açılı (.02-.08), tek kullanımlık bir alettir. WaveOne Gold Glider, Gold ısıl işlemi görmüş, değişken konik açılı (.02-.06), resiprokasyon hareketi ile çalışan ve kanalda vidalama etkisi daha düşük olan tek kullanımlık bir rehber yol aletidir.?

Bu çalışmanın amacl, şekillendirilmesi klinik olarak zor olan S şeklindeki yapay kanallar üzerinde, rotasyon ve resiprokasyon hareketleriyle çalışan rehber aletler varlığı ve yokluğunda WaveOne Gold sisteminin şekillendirme etkinliğinin karşılaştırılmasıdır.

\section{GEREÇ ve YÖNTEM}

Çalışmada 45 adet S-kanallı, 0.02 koniklik açılı, $17 \mathrm{~mm}$ uzunluğunda rezin blok (Endo Training Bloc-S ISO 15, Dentsply Maillefer) kullanılmıştır.

Bloklar numaralandırılmış ve yapay kanallar enjektör yardımıyla siyah mürekkeple boyanmıştır (Pelikan, Hannover, Almanya). Şekillendirme öncesi bloklar bilgisayarda tarayıcıya yerleştirilerek (Epson Perfection V33 Scanner, Seiko Epson Corporation, Tokyo, Japonya) tarama işlemi yapılmış ve görüntüler JPEG formatında kaydedilmiştir. Sonrasında yapay kanallardaki siyah boya, distile su ile yıkanarak temizlenmiş ve rezin bloklar 3 gruba $(n=15)$ ayrılarak aşağıda anlatıldığı gibi şekillendirilmişlerdir:

Grup A: WaveOne Gold (Primary, 25/.07)

Kanal açıklıkları ve çalışma boyu 10 numara K-tipi eğe (Dentsply Maillefer) ile kontrol edilmiştir. WaveOne Gold Primary aleti, üreticilerin tavsiyesi doğrultusunda, X Smart Plus tork kontrollü endodontik motorun (Dentsply Maillefer) içinde yüklü olan WaveOne Gold Primary seçeneği, resiprokasyon hareketiyle $\left(150^{\circ}\right.$ saat yönünün tersine, $30^{\circ}$ saat yönünde) kullanılmıştır. Wave One Gold Primary aleti öncelikle kanalların kuronal bölgesinde $3 \mathrm{~mm}$ 'lik haffi ileri-geri hareketlerle uygulanmıştır. Her 3 mm'lik ilerlemeyi takiben kanal aleti kanaldan çıkarılıp üzerinde biriken debris temizlenmiş ve alet yüzeyi deformasyon varlığı açısından gözle kontrol edilmiştir. Arada 10 numara kanal eğesi ile rekapitülasyon ve irrigasyon yapılıp 
işlem çalışma boyuna ulaşana kadar tekrarlanmıştır.

Grup B: ProGlider + WaveOne Gold

Kanal açıklıkları ve çalışma boyu 10 numara K-tipi eğe ile kontrol edilmiştir. X Smart Plus tork kontrollü endodontik motorun içinde yüklü olan ProGlider seçeneği (rotasyon- 300 rpm hız, 2Ncm tork değerinde) ile pasif basınçla çalışma boyuna ulaşana kadar uygulanmıştır. Arada 10 numara kanal eğesi ile rekapitülasyon ve irrigasyon yapılmıştır. Çalışma boyuna ulaşıldıktan sonra şekillendirmeye WaveOne Gold sistemi ile yukarıda anlatıldığı şekilde devam edilmiştir.

\section{Grup C: WaveOne Gold Glider + WaveOne Gold}

Kanal açıklıkları ve çalışma boyu 10 numara K-tipi eğe ile kontrol edilmiştir. X Smart Plus tork kontrollü endodontik motorun içinde yüklü olan WaveOne Gold Glider ayarı (resiprokasyon- $150^{\circ}$ saat yönünün tersine, $30^{\circ}$ saat yönünde) ile pasif basınçla ileri geri birkaç hamle çalışma boyuna ulaşana kadar uygulanmıştır. Arada 10 numara kanal eğesi ile rekapitülasyon ve irrigasyon yapılmıştır. Çalışma boyuna ulaşıldıktan sonra Wave One Gold sistemi yukarıda anlatıldığı şekilde uygulanarak şekillendirmeler tamamlanmıştır.

Bütün işlemler Ni-Ti şekillendirme sistemleri konusunda tecrübeli bir endodontist tarafından yapılmıştır. Her bir kanal aleti sadece bir yapay kanalın şekillendirilmesinde kullanılmıştır. Her şekillendirme aşamasında kanal açıklıkları 10 numara K-tipi eğe (Dentsply Maillefer) ile kontrol edilmiş ve aletler arasında $1 \mathrm{ml}$ distile su ile irrigasyon yapılmıştır.

Bütün bloklar şekillendirme sonrası kırmızı mürekkep ile boyanmış ve tekrar tarayıcıda taranmıştır. Görüntüler JPEG formatında kaydedilmiştir. Yapay kanalların işlem öncesi ve sonrası görüntüleri imaj analiz programı yardımıyla (Adobe Photoshop CS6, San Jose, CA, ABD) şeffaflaştırııı üst üste çakıștırıımıştır (Resim 1).

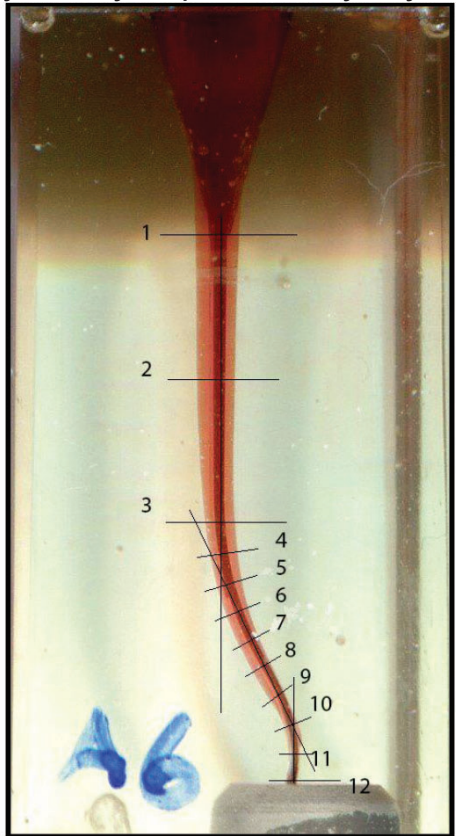

Resim 1. Çakıștırılmış görüntü üzerinde belirlenmiş 12 ölçüm noktasının örnek görüntüsü.
Elde edilen görüntüler üzerinde yapay kanalların uzun aksında dik olacak şekilde 12 ölçüm noktası Madureira ve ark.'nın ${ }^{14}$ açıkladığı yöntemle belirlenmiştir. Öncelikle 4 nokta işaretlenmiştir bunlar: 1. kanal başlangıcl; 3. Düz olan kuronal bölgenin sonu ve ilk eğimin başlangıç noktası; 2. 1.ve 3. noktaların orta noktasl; 12. çalışma boyu. Sonrasında 3. Ve 12. Nokta arasında birbirine eşit uzaklıkta 7 nokta belirlenmiştir bunlar 4., 5., 6., 7., 8., 9., 10. olarak numaralandırılmıştır. 10 ile 12. noktanın ortasına da 11. nokta işaretlenmiştir (Resim 2).

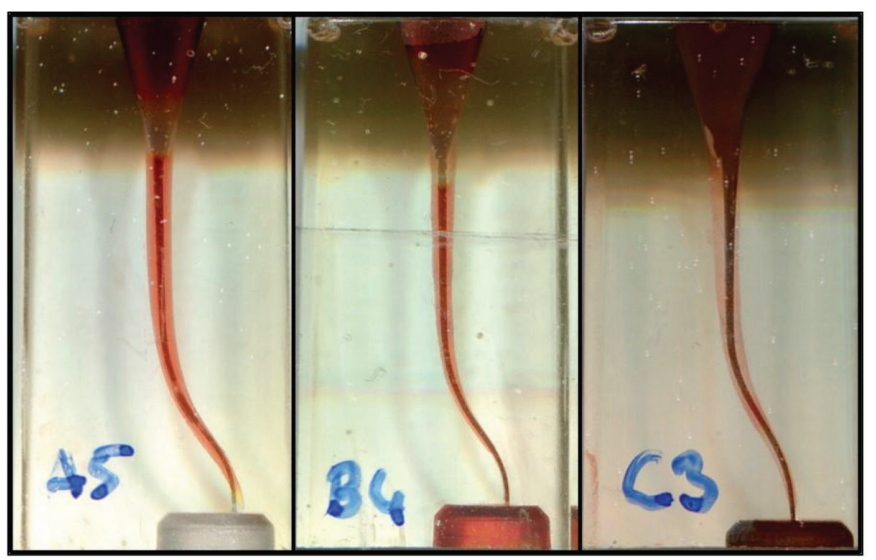

Resim 2. Şekillendirilme öncesi ve sonrası çakıştırılarak elde edilmiş görüntü örnekleri.

Son olarak noktalar 3 ana bölge olarak

değerlendirilmiştir, düz kuronal bölge (1.-3. noktalar ve arası), birinci eğim bölgesi (4.-7. noktalar ve arası) ve apikal eğim bölgesi (8.-12. noktalar ve arası).

Yapay kanalların iç ve dış bölgesinden kaldırılan rezin miktarları çakıştırılan görüntüler üzerinden \%150 büyütmeyle Image $\mathrm{J}$ 1.46r bilgisayar programı (National Institutes of Health, Bethesda, MA, ABD) yardımıyla ölçülmüştür.

Grupların şekillendirme etkinlikleri kanal transportasyonu ve merkezleme oranları açısından karşılaştırılmıştır.

Transportasyon değerleri, kanalın aynı ölçüm noktası üzerinden geçen çizgiden, iç ve dış taraftan kaldırılan rezin miktarlarının birbirinden çıkartııp mutlak değerinin alınmasıyla hesaplanmıştır.

Merkezleme oranı değerleri, kanalın aynı ölçüm noktası üzerinden geçen çizgi üzerinden ölçülen iç ve dış taraftan kaldırılan rezin miktarlarından, dar olan tarafın ölçüm değerinin geniş olan tarafınkine bölünmesiyle hesaplanmiştır.

Bu çalışmada elde edilen verilerin analizi NCSS (Number Cruncher Statistical System) 2007 Statistical Software (NCSS, LLC, Utah, USA) paket programı ile yapılmıştır. Verilerin değerlendirilmesinde tanımlayıcı istatistiksel metodların (ortalama, standart sapma, median, interquartil range) yanı sıra Shapiro-Wilk normallik testi ile değişkenlerin dağılımına bakılmış, normal dağıım göstermeyen değişkenlerin gruplar arası karşılaştırmalarında Kruskal Wallis testi, alt grup karşılaştırmalarında Dunn's çoklu karşılaştırma testi kullanılmıştır. Sonuçlar, $p<0,05$ anlamlılık düzeyinde değerlendirilmiştir. 


\section{BULGULAR}

Gruplar için transportasyon değerleri karşılaştırıldığında, düz kuronal $(p=0,176)$ ve birinci eğim $(p=0,071)$ bölgesinde istatistiksel olarak gruplar arasında anlamlı fark bulunamıştır (Tablo 1).

Tablo 1. Grupların bölgelere göre transportasyon değerleri.

\begin{tabular}{|c|c|c|c|c|c|}
\hline \multicolumn{2}{|c|}{ Transportasyon } & \multirow{2}{*}{$\begin{array}{c}\begin{array}{c}\text { Grup A } \\
\text { WaveOne } \\
\text { Gold } \\
(\mathbf{n}=15)\end{array} \\
3,48 \pm 2,51\end{array}$} & \multirow{2}{*}{$\begin{array}{c}\text { Grup B } \\
\text { ProGlider- } \\
\text { WaveOne } \\
\text { Gold (n=15) }\end{array}$} & \multirow{2}{*}{$\begin{array}{c}\text { Grup C } \\
\text { WaveOne Gold } \\
\text { Glider - } \\
\text { WaveOne Gold } \\
\text { (n=15) } \\
2,49 \pm 1,62\end{array}$} & $\mathbf{p}$ \\
\hline Düz & Ort + SS & & & & \multirow[b]{2}{*}{0,176} \\
\hline $\begin{array}{c}\text { Kuronal } \\
\text { Bölge }\end{array}$ & $\begin{array}{c}\text { Median } \\
\text { (IQR) }\end{array}$ & $3,47(0,53-5,7)$ & $\begin{array}{c}1,33(0,67- \\
3,53)\end{array}$ & $2,5(1-3,57)$ & \\
\hline \multirow{2}{*}{$\begin{array}{l}\text { Birinci } \\
\text { Eğim } \\
\text { Bölgesi }\end{array}$} & Ort \pm SS & $3,16 \pm 1,6$ & $2,95 \pm 3,42$ & $1,88 \pm 1,81$ & \multirow{2}{*}{0,071} \\
\hline & $\begin{array}{c}\text { Median } \\
(\mathrm{IQR})\end{array}$ & $\begin{array}{c}3,23(2,03- \\
3,85)\end{array}$ & $1,75(0,6-4,43)$ & $1,33(0,63-3,05)$ & \\
\hline \multirow{2}{*}{$\begin{array}{l}\text { Apikal } \\
\text { Eğim } \\
\text { Bölgesi }\end{array}$} & Ort \pm SS & $27,26 \pm 19,21$ & $7,09 \pm 9,33$ & $8,99 \pm 6,49$ & \multirow[b]{2}{*}{$\mathbf{0 , 0 0 3}$} \\
\hline & $\begin{array}{c}\text { Median } \\
\text { (IQR) }\end{array}$ & $24(7,9-44,5)$ & $1,3(0,3-10,5)$ & $6,6(5,8-15,1)$ & \\
\hline
\end{tabular}

Kruskal Wallis testi $(* p<0,01)$

Apikal eğim bölgesinde ise $B$ ve $C$ grupları arasında anlamlı farklılık gözlenemezken $(p=0,340)$ A grubunun transportasyon değerleri $B$ ve $C$ grubundan istatistiksel olarak anlamlı yüksek bulunmuştur ( $p=0,002, p=0,011)$. Grupların merkezleme değerleri karşılaştırıldığında, düz kuronal $(p=0,170)$ ve birinci eğim $(p=0,266)$ bölgeleri değerleri arasında anlamlı bir fark bulunamamıştır. (Tablo 2).

Tablo 2. Grupların bölgelere göre merkezleme oranları değerleri.

\begin{tabular}{|c|c|c|c|c|c|}
\hline \multicolumn{2}{|c|}{ Merkezleme Oranı } & \multirow{2}{*}{$\begin{array}{c}\begin{array}{c}\text { Grup A } \\
\text { WaveOne } \\
\text { Gold } \\
(\mathbf{n}=15)\end{array} \\
0,73 \pm 0,09\end{array}$} & \multirow{2}{*}{$\begin{array}{c}\begin{array}{c}\text { Grup B } \\
\text { ProGlider- } \\
\text { WaveOne } \\
\text { Gold (n=15) }\end{array} \\
0,65 \pm 0,12\end{array}$} & \multirow{2}{*}{$\begin{array}{c}\text { Grup C } \\
\text { WaveOne Gold } \\
\text { Glider - } \\
\text { WaveOne Gold } \\
(\mathbf{n}=\mathbf{1 5}) \\
0,71 \pm 0,12\end{array}$} & \multirow{2}{*}{$\mathbf{p}$} \\
\hline \multirow{2}{*}{$\begin{array}{c}\text { Düz } \\
\text { Kuronal } \\
\text { Bölge }\end{array}$} & Ort \pm SS & & & & \\
\hline & $\begin{array}{l}\text { Median } \\
\text { (IQR) }\end{array}$ & $\begin{array}{c}0,74(0,64- \\
0,82)\end{array}$ & $\begin{array}{c}0,63(0,58- \\
0,75)\end{array}$ & $0,69(0,6-0,84)$ & 0,170 \\
\hline \multirow{2}{*}{$\begin{array}{l}\text { Birinci } \\
\text { Eğim } \\
\text { Bölgesi }\end{array}$} & Ort \pm SS & $0,62 \pm 0,18$ & $0,57 \pm 0,10$ & $0,61 \pm 0,22$ & \multirow{2}{*}{0,266} \\
\hline & $\begin{array}{l}\text { Median } \\
\text { (IQR) }\end{array}$ & $0,66(0,43-0,8)$ & $\begin{array}{c}0,62(0,45- \\
0,65)\end{array}$ & $0,69(0,56-0,73)$ & \\
\hline \multirow{2}{*}{$\begin{array}{l}\text { Apikal } \\
\text { Eğim } \\
\text { Bölgesi }\end{array}$} & Ort \pm SS & $1,47 \pm 0,81$ & $1,42 \pm 1,51$ & $3,44 \pm 1,52$ & \multirow[b]{2}{*}{$0,001^{*}$} \\
\hline & $\begin{array}{l}\text { Median } \\
\text { (IQR) }\end{array}$ & $1,5(1,18-1,98)$ & $0,92(0,04-3,3)$ & $4,04(2,95-4,57)$ & \\
\hline
\end{tabular}

Kruskal Wallis testi $(* p<0,01)$

Apikal eğim bölgesinde ise A ve B grupları arasında anlamlı farklılık yokken ( $p=0,395), C$ grubunun merkezleme değerleri $A$ ve $B$ grubunun değerlerinden istatistiksel olarak anlamlı yüksek bulunmuştur ( $p=0,001, p=0,001)$.

\section{TARTIŞMA}

Kök kanalı şekillendirilmesindeki temel hedeflerden biri, orijinal kök kanal anatomisini korumaktır.,15 Bununla birlikte, eğri kök kanallarında transportasyon gibi şekillendirme hataları meydana gelebilmektedir. ${ }^{16}$ Çeşitli çalışmalarda el aletleri ya da diğer mekanik sistemlerle rehber yol oluşturulmasının $\mathrm{Ni}-\mathrm{Ti}$ sistemlerin daha güvenli kullanımını sağladığı gösterilmiştir. ${ }^{17,18}$ Rehber yol oluşturulmasının, transportasyon riskini azalttığını gösteren çalışmalar mevcuttur. ${ }^{19,20}$ Bu çalışmada WaveOne Gold sisteminin şekillendirme etkinliği transportasyon ve merkezleme oranı açısından, farklı rehber yol sistemleri varlığı ve yokluğunda, rezin bloklar üzerinde karşılaştırılarak değerlendirilmiştir.

Rezin bloklar çalışmalarda, şekillendirme işleminin etkinliğinin karşılaştırılması amacıyla, şekillendirme işlemi öncesi ve sonrası görüntülerin çakıştırılması yöntemiyle kullanılmıştır. ${ }^{10,20-24}$ Bu blokların, sistemlerin şekillendirme sonuçlarını ve performansını değerlendirmede standart koşullar sağlamakla birlikte, mikro sertliğinin dentinden farklı olması ve şekillendirme sırasında meydana gelen ısı ile yumuşayıp aletlerin bıçak yüzeyine tutunma gibi bazı dezavantajları da vardır. ${ }^{24}$ Bu çalışmada da kanal boyunca oluşan değişimlerin doğrudan görsel olarak karşılaştıııması amacıyla rezin blokların kullanılması tercih edilmiștir.

Resiprokasyon yapan $\mathrm{Ni}$-Ti sistemlerle şekillendirme öncesi rehber yol oluşturulmasının apikal bölgede daha merkezi şekillendirmeler meydana getirdiğini gösteren çalışmaların yanında, ${ }^{23,25}$ rehber yol oluşturmanın merkezleme oranına ${ }^{26}$ ya da kanalın düzleşmesine etkisi olmadığını gösteren çalışmalar da mevcuttur. Berutti ve ark.'nın ${ }^{11}$ yapay kanallar üzerinde WaveOne'ın (Dentsply, Maillefer) rehber yol varlığı ve yokluğunda etkinliğini kanal eğimi ve kanal aksı üzerinde değerlendirdiği çalışmasında, rehber yol varlığının kanal modifikasyonlarını belirgin şekilde azalttığını göstermişlerdir. Nazorimoghadam ve ark. ${ }^{12}$ resiprokasyon yapan şekillendirme sistemi öncesi rehber yol oluşturulmasının apikal bölgede transportasyonu azalttığını bildirmişlerdir. Bu veriler bu çalışmada rehber yol oluşturulan gruplarda, rehber yol olmayan gruba göre daha az transportasyon meydana gelmesi bulgusuyla uyumlu gözükmektedir.

Rehber yol varlığında WaveOne sistemiyle apikal bölgede daha merkezi şekillendirmeler oluşturulduğu çalışmalarda gösterilmiştir. ${ }^{23,25}$ Bu çalışmada apikal bölgede WaveOne Gold Glider ile oluşturulan rehber yol varlığında merkezleme oranları daha başarılıyken, ProGlider grubuyla rehber yolun olmadığı grupta anlamlı bir fark oluşmamıştır. Aydın ve ark. ${ }^{27}$ farklı rehber yol aletlerinin şekillendirme etkinliklerini çekilmiş dişlerde orta derece eğimli kök kanallarında mikro bilgisayarlı tomografiyle karşılaştırdıkları çalışmalarında, WaveOne Gold Glider'ın merkezleme oranının ProGlider'dan daha başarıı olduğunu göstermişlerdir. Bu bulgu bu çalışmadaki WaveOne Gold Glider grubunun, ProGlider grubundan apikal bölgede daha merkezi oranlar vermesini desteklemektedir. Transportasyon değerleri karşılaştırıldığında, yine bu çalışmanın sonuçlarıyla uyumlu şekilde resiprokasyon yapan WaveOne Gold Glider ve rotasyon yapan ProGlider rehber yol sistemleri arasında anlamlı bir fark bildirilmemiştir.

\section{SONUÇLAR}

Bu çalışmanın koşulları altında değerlendirildiğinde, WaneOne Gold sistemiyle S kanalların şekillendirilmesinde öncelikle resiprokasyon yapan sistem Wave- 
One Gold Glider ile giriş yolu oluşturulduğunda apikal bölgede daha merkezi bir șekillendirme elde edilmektedir. Wave One Gold sistemi öncesi rehber yol oluşturmak apikal bölgedeki transportasyon miktarını azaltmaktadır.

\section{KAYNAKLAR}

1. Peters OA. Current challenges and concepts in the preparation of root canal systems: a review. J Endod 2004; 30: 559-567.

2. Hulsmann M, Peters OA, Dummer PMH. Mechanical preparation of root canals: shaping goals, techniques and means. Endod Top 2005; 10: 30-76.

3. Plotino G, Ahmed HM, Grande NM, Cohen S, Bukiet

F. Current assessment of reciprocation in endodontic preparation: A comprehensive review - Part II: Properties and effectiveness. J Endod 2015; 41: 1939-1950.

4. Varghese NO, Pillai R, Sujathen UN, Sainudeen S, Antony $\mathrm{A}$, ve ark. Resistance to torsional failure and cyclic fatigue resistance of ProTaper Next, WaveOne, and Mtwo files in continuous and reciprocating motion: An in vitro study. J Conserv Dent 2016; 19: 225-230.

5. Dhingra A, Kochar R, Banerjee S, Srivastava P. Comparative evaluation of the canal curvature modifications after instrumentation with one shape rotary and Wave One reciprocating files. J Conserv Dent 2014; 17 :138-141.

6. Roane JB, Sabala C. Clockwise or counterclockwise. J Endod 1984; 10: 349-353.

7. Gavini G, Santos MD, Caldeira CL, Machado MEL, Freire LG ve ark. Nickel-titanium instruments in endodontics: a concise review of the state of the art. Braz Oral Res. 2018; 32: e67.

8. Gagliardi J, Versiani MA, de Sousa-Neto MD, Plazas-Garzon A, Basrani B. Evaluation of the shaping characteristics of ProTaper Gold, ProTaper NEXT, and ProTaper universal in curved canals. J Endod. 2015; 41: 1718-1724.

9. Kirchhoff AL, Chu R, Mello I, Garzon AD, dos Santos $M$ ve ark. Glide path management with single- and multiple-instrument rotary systems in curved canals: a micro-computed tomographic study. J Endod 2015; 41: 1880-1883.

10. Berutti E, Cantatore G, Castellucci A, Chiandussi G, Pera F ve ark. Use of nickel-titanium rotary PathFile to create the glide path: comparison with manual preflaring in simulated root canals. J Endod 2009; 35: 408-412.

11. Berutti E, Paolino DS, Chiandussi G, Alovisi M, Cantatore $G$ ve ark. Root canal anatomy preservation of WaveOne reciprocating files with or without glide path. J Endod 2012; 38: 101-104.

12. Nazarimoghadam $K$, Daryaeian $M$, Ramazani $N$. An in vitro comparison of root canal transportation by reciproc file with and without glide path. J Dent (Tehran) 2014; 11: 554-559.

13. Topçuoğlu HS, Düzgün $S$, Aktı A, Topçuoğlu G. Laboratory comparison of cyclic fatigue resistance of Wave-
One Gold, Reciproc and WaveOne files in canals with a double curvature. Int Endod J 2017; 50: 713-717.

14. Madureira RG, Forner Navarro L, Llena MC, Costa M. Shaping ability of nickel-titanium rotary instruments in simulated S-shaped root canals. Oral Surg Oral Med Oral Pathol Oral Radiol Endod 2010; 109: e136-144.

15. Thompson SA, Dummer PM. Shaping ability of Hero 642 rotary nickel-titanium instruments in simulated root canals: Part 2. Int Endod J 2000; 33: 255-261.

16. Hülsmann M, Stryga F. Comparison of root canal preparation using different automated devices and hand instrumentation. J Endod 1993; 19: 141-145.

17. Patiño PV, Biedma BM, Liébana $C R$, Cantatore $G$, Bahillo JG. The influence of a manual glide path on the separation rate of NiTi rotary instruments. J Endod 2005; 31: 114-116.

18. Berutti E, Negro AR, Lendini M, Pasqualini D. Influence of manual preflaring and torque on the failure rate of ProTaper rotary instruments. J Endod 2004; 30: 228-230. 19. Peters $\mathrm{OA}$, Peters $\mathrm{Cl}$, Schönenberger $\mathrm{K}$, Barbakow $\mathrm{F}$. ProTaper rotary root canal preparation: assessment of torque and force in relation to canal anatomy. Int Endod J 2003; 36: 93-99.

20. Bonaccorso A, Cantatore G, Condorelli GG, Schäfer E, Tripi TR. Shaping ability of four nickel-titanium rotary instruments in simulated S-shaped canals. J Endod 2009; 35: 883-886.

21. Ding-Ming $H$, Hong-Xia $L$, Cheung GS, Lan Z, Hong $T$ ve ark. Study of the progressive changes in canal shape after using different instruments by hand in simulated S-shaped canals. J Endod 2007; 33: 986-989.

22. Ersev H, Yilmaz B, Ciftçioglu E, Ozkarsli SF. A comparison of the shaping effects of 5 nickel-titanium rotary instruments in simulated S-shaped canals. Oral Surg Oral Med Oral Pathol Oral Radiol Endod 2010; 109: e86-93.

23. Yilmaz A, Kucukay ES, Istektepe $M$, Sisli SN, Ersev H ve ark. Comparison of the Shaping Ability of WaveOne Reciprocating Files with or without Glide Path in Simulated Curved S-shaped Root Canals. J Int Soc Prev Community Dent. 2017; 7: 13-17.

24. Zhang $L$, Luo HX, Zhou XD, Tan $H$, Huang DM. The shaping effect of the combination of two rotary nickel-titanium instruments in simulated S-shaped canals. J Endod 2008; 34: 456-458.

25. Lim YJ, Park SJ, Kim HC, Min KS. Comparison of the centering ability of Wave-One and Reciproc nickel-titanium instruments in simulated curved canals. Restor Dent Endod 2013; 38: 21-25.

26. Coelho MS, Fontana CE, Kato AS, de Martin AS, da Silveira Bueno CE. Effects of glide path on the centering ability and preparation time of two reciprocating instruments. Iran Endod J 2016; 11: 33-37.

27. Aydın ZU, Keskin NB, Özyürek T, Geneci F, Ocak M 
ve ark. Microcomputed assessment of transportation, centering ratio, canal area, and volume increase after single-file rotary and reciprocating glide path instrumentation in curved root canals: a laboratory study. J Endod 2019; 45: 791-796. 